\title{
Measurement and simulation of the background in the CMS muon detectors
}

\author{
Cesare Calabria ${ }^{1,2, *}$ on behalf of the CMS Collaboration \\ ${ }^{1}$ INFN (Bari, Italy) \\ ${ }^{2}$ CERN (Geneva, Switzerland)
}

\begin{abstract}
The study of the different backgrounds which the muon detectors of the CMS experiment are exposed to, is fundamental to assess the system longevity and project its performance to the conditions expected for HL-LHC. In this respect, an accurate modelling of the backgrounds in simulation is of prime importance as many studies rely on simulation-based predictions while these future conditions have never been experienced in reality. This paper will present the state of the art of the work carried out to understand backgrounds observed with data collected during the LHC runs, as well as at the CERN highintensity gamma irradiation facility. Furthermore, the effort made to improve the accuracy of FLUKA and GEANT4 based simulations of background will be thoroughly described.
\end{abstract}

\section{Introduction}

The High Luminosity LHC (HL-LHC) [1] upgrade is planned to extend the sensitivity for new physics searches by increasing the instantaneous luminosity up to $5 \times 10^{34} \mathrm{~cm}^{-2} \mathrm{~s}^{-1}$ $\left(7.5 \times 10^{34} \mathrm{~cm}^{-2} \mathrm{~s}^{-1}\right.$ in the ultimate scenario) and the centre-of-mass energy for proton-proton $(\mathrm{p}-\mathrm{p})$ collisions to $14 \mathrm{TeV}$. The average number of pileup interactions per bunch-crossing is expected to reach 140 (200 in the ultimate scenario). The integrated luminosity will increase tenfold with respect to the original design values, from $300 \mathrm{fb}^{-1}$ for "Phase-1" (the current LHC period) to $3000 \mathrm{fb}^{-1}$ in the coming two decades, for both the CMS and ATLAS detectors. The projected evolution of instantaneous and integrated luminosities and pile-up with time is shown in Fig. 1 for the design scheme. As it can be seen from the figure, the current "Phase-1" data taking period will end in the year 2023, followed by a shutdown for the HL-LHC upgrade ending in 2026. The high luminosity data taking period with the upgraded LHC is called "Phase-2" and is expected to last from 2026 till 2038.

The high rate expected at HL-LHC is a major challenge for the particle muon detector longevity and the muon reconstruction. In fact it can decrease the gas gain and increase hitefficiency losses. Moreover, radiation damage can lead to noisier electronics performance and even fatal failures of entire electronic boards. Thus, simulation and study of the background in the muon system is of primary importance for future operations.

*e-mail: cesare.calabria@ba.infn.it 


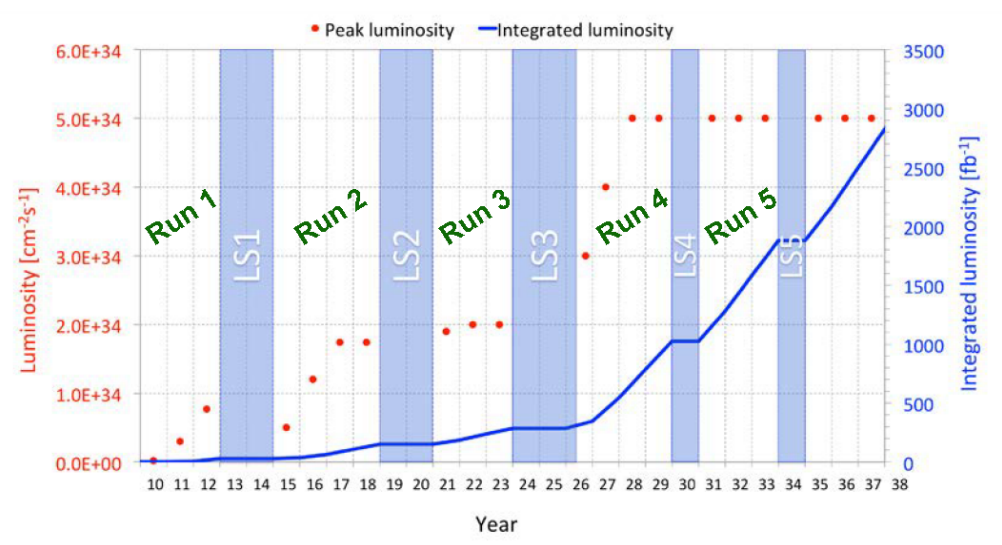

Figure 1. The design LHC schedule and values for instantaneous and integrated luminosities[2].

\section{The CMS muon system and its upgrade}

A schematic view of one R-z quadrant of the CMS detector after Phase-2 upgrades is shown in Fig. 2, where the location of the muon chambers is highlighted (details in Ref. [3]). In the CMS experiment muons are detected with multi-layer gas-ionization detectors positioned outside the solenoid. As of 2017, three types of chambers make up the CMS muon system: Drift Tube (DT) chambers, Cathode Strip Chambers (CSC) and Resistive Plate Chambers (RPC) (for details see Ref. [3]). The DT and CSC chambers are located in the pseudorapidity regions $|\eta|<1.2$ and $0.9<|\eta|<2.4$, respectively, and are complemented by the RPC detectors in both barrel and endcaps, with a maximum reaching $|\eta|=1.9$. The active chamber areas are arranged to overlap, avoiding gaps in the $\eta$ coverage. The CSC and RPC chambers overlap also in $\phi$. The DTs are segmented in long aluminum drift cells. The position of a traversing muon is determined by measuring the drift time to the anode wire in the center of each cell, with an optimally shaped electric field. The spatial resolution per cell is $250 \mu \mathrm{m}$ or better, which translates into about $100 \mu \mathrm{m}$ resolution per 8-layer chamber. The CSCs operate as standard multi-wire proportional counters with a finely segmented cathode strip readout. The strips run radially outward to measure the muon position in the bending plane, while the anode wires provide a measurement in $\mathrm{R}$. The precise position in the dimension orthogonal to the strips can be reconstructed by interpolating the charges read out on the strips. The CSC detection technology was chosen because it provides good position and time resolutions of hits along a muon trajectory, can handle high particle rates, and is able to operate in strong non-uniform magnetic fields. Typical resolution figures for position and time are 50-140 $\mathrm{mm}$ (depending on chamber type) and 3 ns per chamber, respectively. The RPCs are doublegap chambers operated in avalanche mode, at high electric field. They use High Pressure Laminate (commonly known as bakelite) electrodes with a high bulk resistivity. RPCs are mainly used for accurate timing and fast triggering, with an excellent intrinsic resolution of about $1.5 \mathrm{~ns}$ for a double-gap chamber. This allows in particular the identification of the corresponding bunch crossing.

The existing muon detectors are all essential for identifying and measuring muons with the required precision and accuracy needed to fulfil the CMS physics program. All muon subsystems worked with high efficiency and resolution even at $1.7 \times 10^{34} \mathrm{~cm}^{-2} \mathrm{~s}^{-1}$, the highest instantaneous luminosity reached by the summer of 2017. In order to maintain the present excellent performance of the CMS muon system (see Ref. [4]) at high luminosity within a 


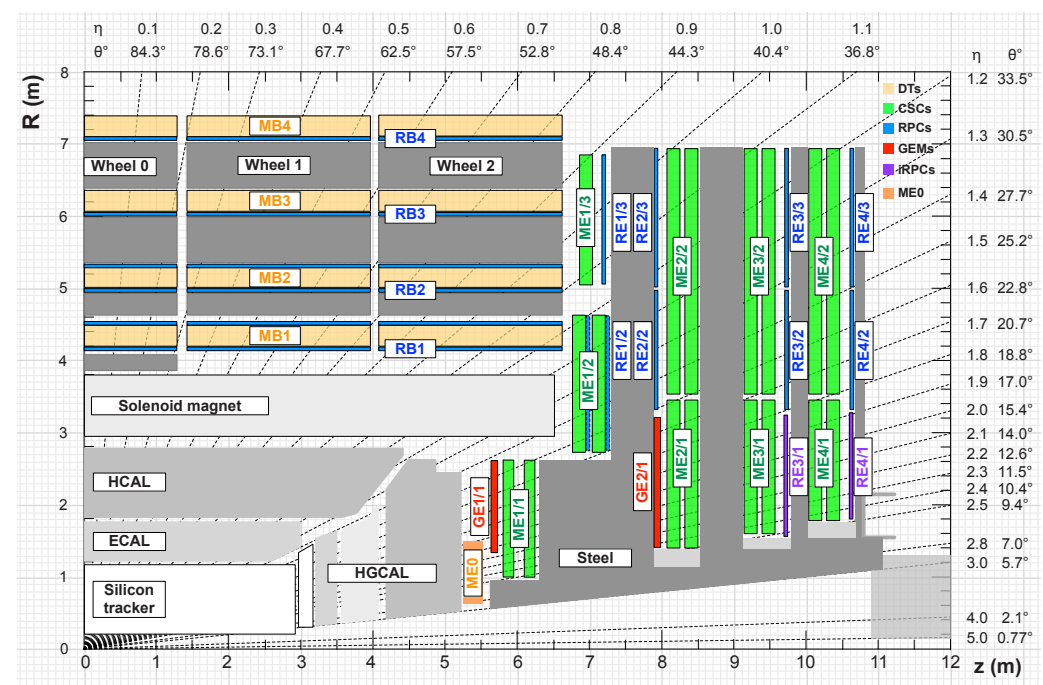

Figure 2. An R-z cross section of a quadrant of the CMS detector, including the Phase-2 upgrades (RE3/1, RE4/1, GE1/1, GE2/1, ME0).

large geometrical acceptance, several measures are being undertaken such as: the replacement of the existing detector electronics, increasing the acceptance and enhancing the muon trigger and reconstruction capabilities at high $\eta$. In particular the last point is achieved with new forward muon detectors (GE1/1, GE2/1, ME0, RE3/1, and RE4/1 ${ }^{1}$ ) covering the $\eta$ range up to 2.8 , providing further high resolution measurement points and significantly increasing the lever arm for the local track reconstruction (details in Ref. [2]).

\section{Background in the CMS muon system}

Background simulation is mandatory to cope with the harsh conditions never faced before at LHC, and design properly the electronics and the new detectors. Neutron induced background is the main source of background in the CMS muon system. Neutrons (both fast and thermal) induce background hits via nuclear interactions and capture, followed by gamma emission of $\mathrm{O}(0.5-10 \mathrm{MeV})$ and (mainly) Compton scatter of electrons of $\mathrm{O}(\mathrm{MeV})$ that subsequently ionize the chamber gas (see Fig. 3).

There is a big effort in CMS to establish a detailed simulation using tools like FLUKA [6, 7] and GEANT4 [8-10]. FLUKA is used to simulate the p-p collisions and the transport of particles in the CMS detector (including long living neutrons) and estimate the expected fluxes in the detectors. GEANT4 is used to estimate the probability for these particles to create a spurious signal in the detector (sensitivity). In order to have the highest level of precision, the CMS detector and the cavern geometry have been carefully modelled both in FLUKA and GEANT4. The hit rate expected in the new detectors was assessed by convoluting the fluxes and the sensitivities. One example of the results obtained with this

\footnotetext{
${ }^{1}$ In the CMS naming convention the first letter indicates the technology used: $\mathrm{G}=$ Gas Electron Multiplier (GEM), R = RPC, M = CSC. Though based on a GEM technology, ME0 represents here an exception because of its position in the detector and its 6-layered structure more similar to the CSC design. The second letter indicates the region where the detectors are placed inside $\mathrm{CMS}(\mathrm{B}=$ barrel, $\mathrm{E}=$ endcap). The following two numbers indicate the station and the ring respectively.
} 

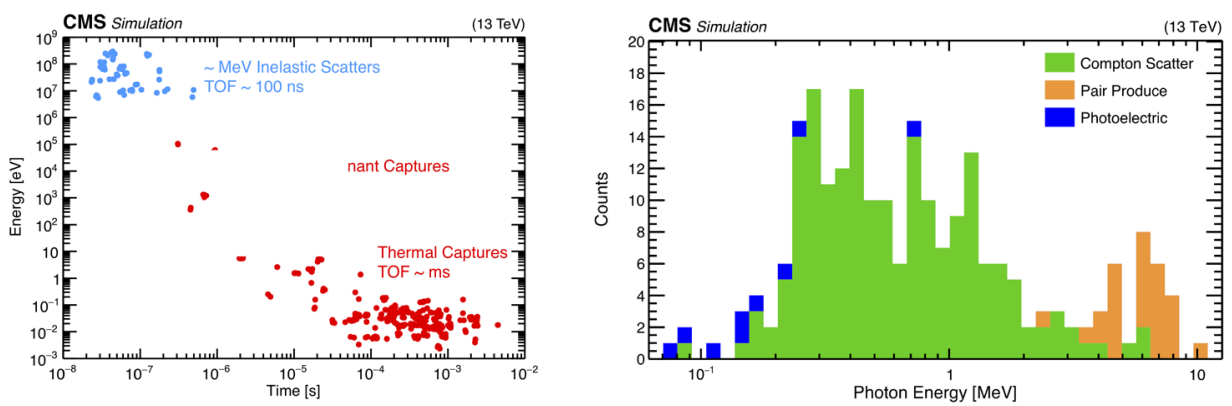

Figure 3. Left plot: final energy of simulated neutron vs. the time of simulated detector hit (measured with respect to the the $\mathrm{p}-\mathrm{p}$ collision) in the CMS muon cathode strip chambers (CSC). Hits are induced by electrons which are produced from photons which are produced from thermal or resonant neutron capture or from neutron inelastic scattering. Red dots indicate simulated hits induced by thermal and resonant neutron captures and blue dots indicate simulated hits induced by neutron inelastic scatters. Right plot: stacked histogram of the final energy of simulated photons at the time they produce simulated electrons that lead to simulated detector hits. The photons are categorized by the process by which the electrons were created or scattered. The most common process by which electrons that eventually lead to simulated hits are formed is Compton scattering. Both plots are made with GEANT4 simulated minimum-bias $\mathrm{p}-\mathrm{p}$ collisions at $13 \mathrm{TeV}$ using the High Precision neutron interaction cross section library and the 2015 CMS simulated geometry.

simulation can be seen in Fig. 4 (left) for the GE1/1 detector. Moreover, a full GEANT4 based Monte Carlo simulation has been also set up to include effects of minimum-bias $\mathrm{p}-\mathrm{p}$ collisions and the neutron background in the CMS analysis framework. To this end the standard CMS GEANT4 configuration has been modified in order to address the long lifetime and low energy of the neutron, by increasing tracking time of all particles and removing all energy thresholds where they existed (see next section).
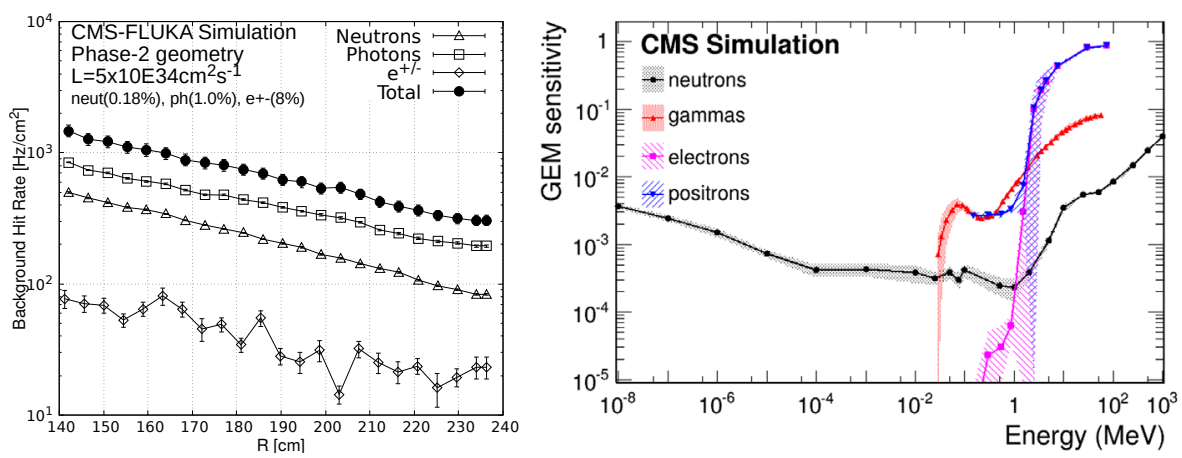

Figure 4. Left plot: estimated background hit rate extracted with a combination of FLUKA+GEANT4 simulations considering CMS-FLUKA Phase- 2 geometry and an instantaneous luminosity of $5 \times 10^{34}$ $\mathrm{cm}^{-2} \mathrm{~s}^{-1}$ for the upgrade GE1/1 station. The plot shows the contribution of the different neutron induced background particles (neutrons, photons, $\mathrm{e}^{+} \mathrm{e}^{-}$) weighted according to detector sensitivity and the total contribution. Right plot: Energy-dependent sensitivity (defined as the probability to produce a measured hit in the chamber) of the GE1/1 chamber to neutrons, photons, electrons, and positrons, as a function of the incident particle energy. 


\section{Neutron background measured in data}

The Muon community is studying the neutron-induced background also in the CMS data collected during Run2. The comparison with the simulation is a fundamental step for validating the simulation technique and to make accurate predictions for HL-LHC.

\subsection{Neutron background measured in the CSC system}

In the CSC community, the impact of neutron hits has been extensively studied[12]. The hits have been selected by looking for $Z \rightarrow \mu \mu$ events and identifying candidate neutron-induced hits by looking away from the triggering muon. In order to distinguish neutrons from out-oftime pileup gaps in the LHC proton bunch structure of at least 35 bunch crossings (BX) have been considered and $\mathrm{p}-\mathrm{p}$ bunch trains with exactly 48 BX to identify $\mathrm{p}-\mathrm{p}$ collisions occurring just after such gaps have been used. Finally the end-of-gap thermal neutron induced hits have been considered and they were compared to thermal-neutron induced hits from simulation. For all CSCs at various $\mathrm{r}$ and $\mathrm{z}$ positions, simulation reproduces data to within a factor of 2 , as it can be seen in Fig. 5 [12].
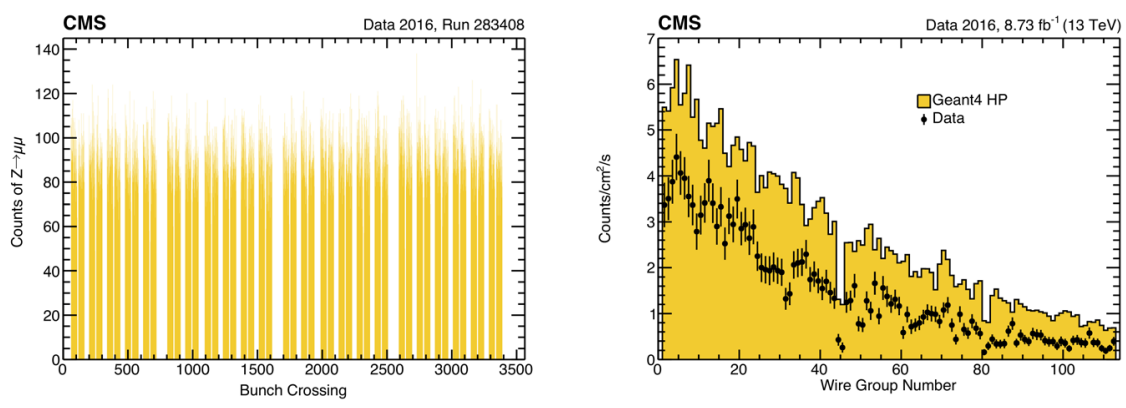

Figure 5. Left plot: Histogram of bunch crossing number in a particular CMS data taking period during LHC proton-proton collisions in 2016, showing the gaps of various sizes in the LHC bunch structure. Right plot: thermal-neutron induced anode wire hits for CMS data collected during LHC p-p collisions in 2016 by the inner ring of the CSC second station (ME2/1) (black points). CMS data are compared to the full GEANT4 simulation (yellow bars) intended to simulate minimum-bias p-p collisions at $13 \mathrm{TeV}$ using the High Precision (HP) neutron interaction cross section library and the 2015 CMS simulated geometry.

\subsection{Neutron background measured in the RPC system}

Background in the cavern has been studied also in terms of RPC hit rate as a function of the instantaneous luminosity[13]. The rates were measured during the $\mathrm{p}-\mathrm{p}$ collision data taking and averaged per run. Also the instantaneous luminosity is averaged per run. The linear dependency allowed an extrapolation of the rates to the HL-LHC regime to be made, as it can be seen in the left plot of Fig. 6. Moreover, the background hit rate measured in data has been compared with the FLUKA simulation at an instantaneous luminosity of $10^{34} \mathrm{~cm}^{-2} \mathrm{~s}^{-1}$. Background simulations reproduce the measured rates within a factor of two in the worst case, see the right plot in Fig. 6 [13]). 

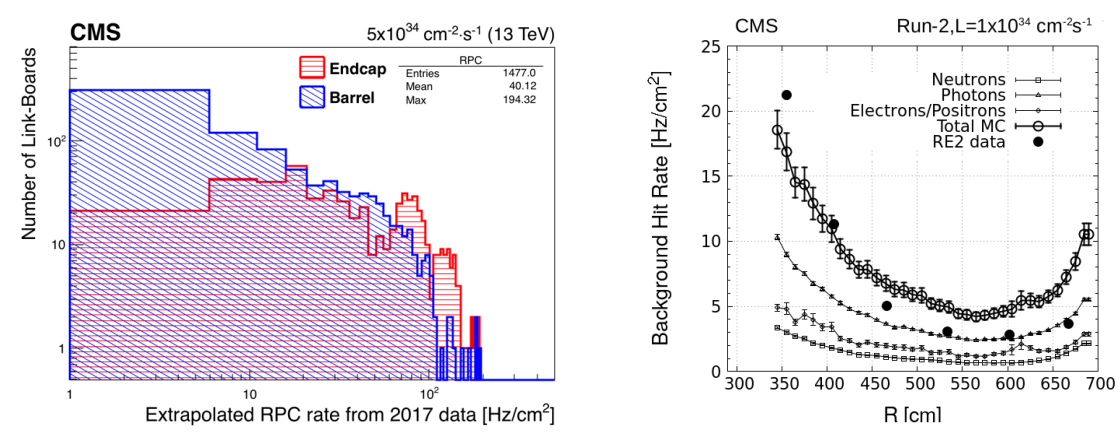

Figure 6. Left plot: expected mean and maximum rate for the entire RPC system from 2017 data extrapolated to the instantaneous luminosity of $5 \times 10^{34} \mathrm{~cm}^{-2} \mathrm{~s}^{-1}$. The maximum expected rate from 2017 data is $200 \mathrm{~Hz} / \mathrm{cm}^{2}$. Right plot: comparison of the background hit rate between FLUKA simulation and RPC measurements for the second RPC endcap station and instantaneous luminosity of $1 \times 10^{34}$ $\mathrm{cm}^{-2} \mathrm{~s}^{-1}$. Simulation results use a Run-2 CMS geometry.

\section{Longevity tests}

Elements of the current system are already 15-20 years old, and eventually may need to last 40 years. Thus existing equipment and new detectors must be certified for HL-LHC, i.e. 6 times the integrated radiation doses and 5 times the signal and background rates with respect to the LHC. To this end accelerated tests are ongoing by exposing detectors and electronics to irradiation sources. Accelerated tests are indicative only, and at least a factor of 3 larger than what we expect to see over the HL-LHC lifetime is considered.

Several chambers are currently under test for all the subsystems at the CERN GIF++ facility [11] that provides $662 \mathrm{keV}$ photons emitted by an intense $14 \mathrm{TBq}{ }^{137} \mathrm{Cs}$ source and a high momentum muon beam $(100 \mathrm{GeV})$. Moreover, neutron-induced photons have an energy in the range $0.1-10 \mathrm{MeV}$; thus, GIF++ provides a fairly realistic simulation of the HL-LHC conditions. Also a muon beam is available and it provides excellent probes for detector performance studies in the presence of high radiation. The tests will continue until the HLLHC total integrated charge (including safety factor) is reached, see Table 1 [2].

Table 1. Integral radiation dose, hit rate and accumulated charge for the CMS muon detectors after

Phase-2 upgrades, at the end of HL-LHC running, assuming an instantaneous luminosity of $5 \times 10^{34} \mathrm{~cm}^{-2} \mathrm{~s}^{-1}$. Only the worst-case values are given. The numbers shown do not include safety factors.

\begin{tabular}{|c||c|c|c|c|c|c|c|}
\hline & DT & CSC & RPC & iRPC & GE1/1 & GE2/1 & ME0 \\
$|\eta|$ range & $0-1.2$ & $0.9-2.4$ & $0-1.9$ & $1.8-2.4$ & $1.6-2.15$ & $1.6-2.4$ & $2.0-2.8$ \\
\hline \hline Hit rate $\left(\mathrm{Hz} / \mathrm{cm}^{2}\right)$ & 50 & 4500 & 200 & 700 & 1500 & 700 & 48000 \\
\hline \hline Charge per wire $(\mathrm{mC} / \mathrm{cm})$ & 20 & 110 & - & - & - & - & - \\
\hline Charge per area $\left(\mathrm{mC} / \mathrm{cm}^{2}\right)$ & - & - & 280 & 330 & 6 & 3 & 280 \\
\hline
\end{tabular}

About $15 \%$ of DT chambers, most exposed to background, saw observable gas gain loss (see the left plot in Fig. 7), but muon reconstruction efficiency will remain high, thanks to multiple layers of DTs on the path of a muon. In addition, ageing mitigation measures are being explored (for instance no gas recirculation, HV adjustment, shielding for chambers, etc.)[2]. Concerning the CSC system, a total integrated charge of 330 (ME1/1) and 340 $(\mathrm{ME} 2 / 1) \mathrm{mC} / \mathrm{cm}$ has been collected and no noticeable gas gain loss has been appreciated up 

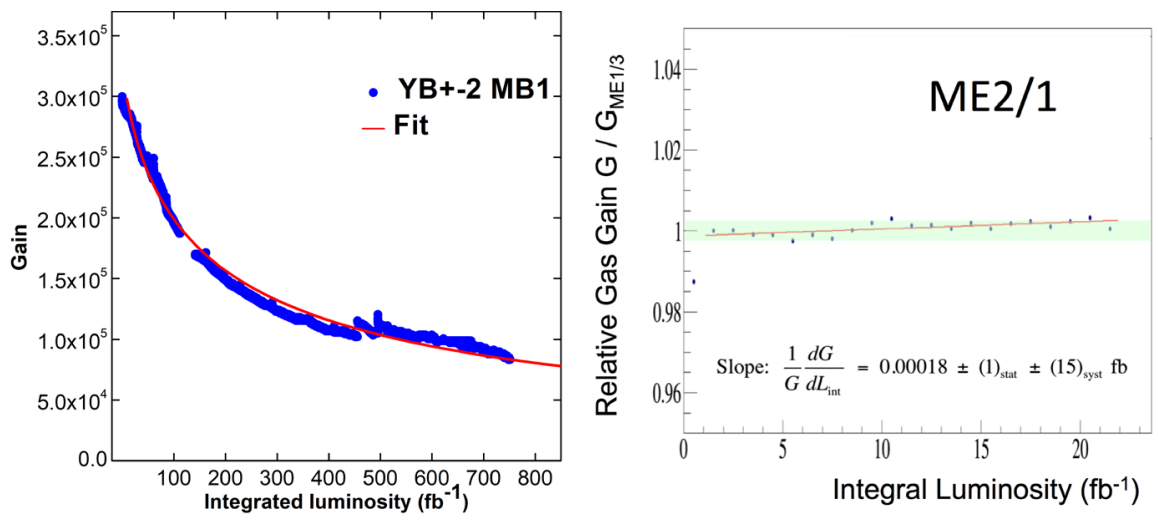

Figure 7. Left plot: Gain evolution, estimated from the measured response of the drift tube chambers, as a function of integrated luminosity for an MB1 chamber of the external wheels. Right plot: average gas gain in CSC ME2/1 chambers normalized to that in ME1/3 as a function of integrated luminosity. The systematic uncertainty in the slope is assessed from the range of small wiggles, indicated by the shaded area $( \pm 0.3 \%)$.

to 3 times the expected charge at HL-LHC[2] (see the right plot in Fig. 7). The tests are still ongoing. No noticeable RPC detector degradation effect was observed up to more than one third of the expected charge at HL-LHC (351 and $160 \mathrm{mC} / \mathrm{cm}^{2}$ on the two chambers under test), see the left plot in Fig. 8. Longevity test recently started also on large size prototype of iRPC $\left(33 \mathrm{mC} / \mathrm{cm}^{2}\right)$, and the main parameters are stable so far. Also in this case the tests are continuing. For the GEM detector a total charge of $125 \mathrm{mC} / \mathrm{cm}^{2}$ has been accumulated so far, corresponding to ten years of GE1/1 (GE2/1) operation at the HL-LHC with a safety factor 21 (42), and $44 \%$ of the total ME0 operation. No ageing effects have been observed[14], see the right plot in Fig. 8. A new ageing study is underway also with an X-ray source on an ME0 chamber prototype. The accumulated total charge is $875 \mathrm{mC} / \mathrm{cm}^{2}$, corresponding to ten years of operation in the ME0 region with a safety factor 3. Also in this case no ageing effects have been observed[14].

\section{Conclusions and outlook}

Within the CMS Muon community a big effort has been made to set up the most accurate neutron background simulation for high luminosity LHC. Nonetheless, work is still ongoing to increase the accuracy of the simulation and to fully understand the neutron background in real data. Longevity tests on the muon chambers are ongoing to re-certify the existing detectors for HL-LHC and prove the capabilities of the new detectors. So far some ageing effects have been observed only in the drift tube system. The tests will continue in the future, especially for those chambers that have not yet reached the HL-LHC total integrated charge.

\section{References}

[1] G. Apollinari, O. Brüning, T. Nakamoto and L. Rossi, "High Luminosity Large Hadron Collider HL-LHC", CERN Yellow Report (2015) no.5, 1.

[2] CMS Collaboration, "The Phase-2 Upgrade of the CMS Muon Detectors", CMS-TDR016 (2017). 

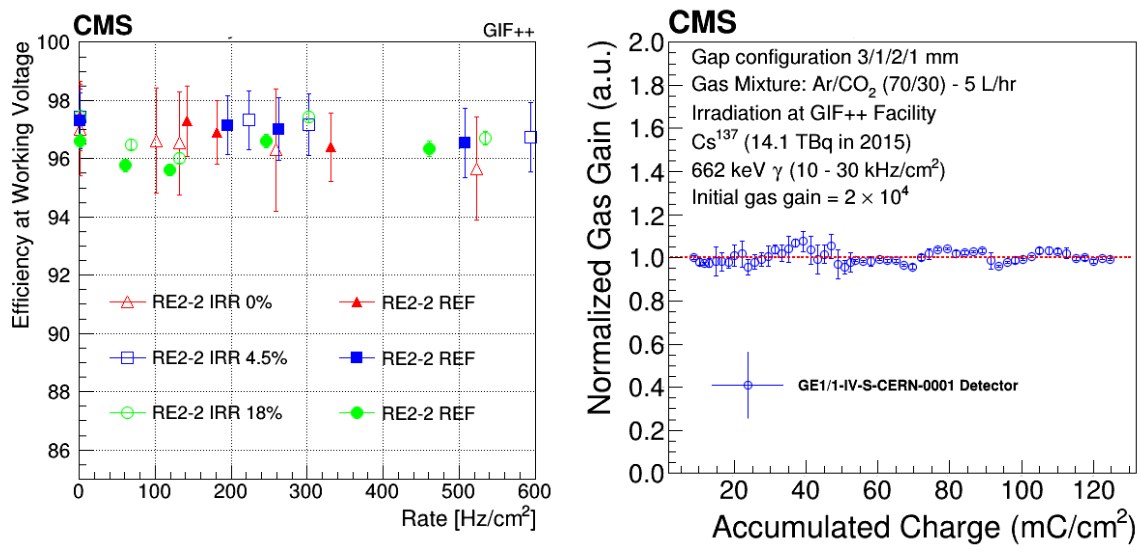

Figure 8. Left plot: Evolution of the hit efficiency at the working point, for RPC RE2/2 chambers as a function of the $\gamma$ rate per unit area. Both irradiated and non-irradiated reference chambers are shown. The measurements have been repeated after different periods of irradiation (corresponding to $18 \%$ and $31 \%$ of the total integrated charge expected by the end of HL-LHC). Right plot: result of the GIF++ Triple-GEM ageing test showing the normalized and corrected effective gas gain as a function of the accumulated charge. The detector under test is a GE1/1-IV-S-CERN-0001 chamber operating in $\mathrm{Ar} / \mathrm{CO}_{2}(70 / 30)$ at an initial gas gain of $2 \times 10^{4}$.

[3] CMS Collaboration, “The CMS experiment at the CERN LHC”, JINST 3 (2008) S08004.

[4] CMS Collaboration, "Performance of the CMS muon detector and muon reconstruction with proton-proton collisions at $\sqrt{s}=13 \mathrm{TeV}$ ", JINST 13 (2008) P06015.

[5] F. Sauli, "GEM: A new concept for electron amplification in gas detectors", Nucl. Instrum. Meth. A 386 (1997) 531.

[6] T.T. Böhlen et al., "The FLUKA Code: Developments and Challenges for High Energy and Medical Applications", Nucl. Data Sheets 120 (2014) 211.

[7] A. Ferrari, P.R. Sala, A. Fassó, and J. Ranft, "FLUKA: a multi-particle transport code", CERN-2005-10 (2005).

[8] GEANT4 Collaboration, "Geant4 - a simulation toolkit", Nucl. Instrum. Meth. A 506 (2003) 250.

[9] J. Allison et al., "Geant4 developments and applications", IEEE Trans. Nucl. Scien. 53 (2006) 270.

[10] J. Allison et al., "Recent developments in Geant4", Nucl. Instrum. Meth. A 835 (2016) 186.

[11] D. Pfeiffer et al., "The Radiation Field in the Gamma Irradiation Facility (GIF++) at CERN", Nucl. Instrum. Meth. A 866 (2017) 91.

[12] A. Dasgupta et al., "Study of neutron-induced background hits in the CMS endcap muon system”, PoS(EPS-HEP2017) 782 (2017).

[13] CMS Collaboration, "RPC Detector Performance Results for 2016 and 2017”, CMS Detector Performance Report CMS-DP-2018-001 (2018).

[14] F. Fallavollita et al., “Triple GEM Ageing Studies”, CMS Document 13528-v1 (2018), https://cms-docdb.cern.ch/cgi-bin/DocDB/ShowDocument?docid=13528. 